\title{
Espessura central e periférica da córnea: influência na medida da pressão intra-ocular pelo Tonopen
}

\author{
Central and peripheral corneal thickness: influence on the IOP measurement \\ by Tonopen
}

\author{
Willer Otávio Guimarães Amaral ${ }^{1}$ \\ Roberto Márcio Batista Teixeira ${ }^{2}$ \\ Leandro Moulin Alencar ${ }^{3}$ \\ Sebastião Cronemberg'er ${ }^{4}$ \\ Nassim Calixto ${ }^{5}$
}

Trabalho realizado no Serviço de Glaucoma do Hospital São Geraldo da Universidade Federal de Minas Gerais - UFMG - Belo Horizonte (MG) - Brasil.

Fellow do Serviço de Glaucoma do Hospital São Geraldo, Hospital das Clínicas da Universidade Federal de Minas Gerais - UFMG - Belo Horizonte (MG) - Brasil.

Médico Oftalmologista e Preceptor do Serviço de Glaucoma do Hospital São Geraldo, Hospital das Clínicas da UFMG - Belo Horizonte (MG) - Brasil.

${ }^{3}$ Fellow do Serviço de Glaucoma do Hospital São Geraldo, Hospital das Clínicas da UFMG - Belo Horizonte (MG) Brasil.

${ }^{4}$ Professor Titular de Oftalmologia da Faculdade de Medicina da UFMG - Belo Horizonte (MG) - Brasil.

Professor Titular de Oftalmologia da Faculdade de Medicina da UFMG - Belo Horizonte (MG) - Brasil.

Os autores não têm interesse comercial nos produtos e equipamentos utilizados neste estudo.

Endereço para correspondência: Rua Martim de Carvalho, 410/501 - Belo Horizonte (MG) CEP 30190-090 E-mail: cronem@task.com.br

Recebido para publicação em 11.06.2005

Versão revisada recebida em 20.10.2005

Aprovação em 31.10.2005

Nota Editorial: Depois de concluída a análise do artigo sob sigilo editorial e com a anuência dos Drs. Simone Finzi e Haroldo Vieira de Moraes Jr. sobre a divulgação de seus nomes como revisores, agradecemos sua participação neste processo.

\begin{tabular}{|c|}
\hline RESUMO \\
\hline 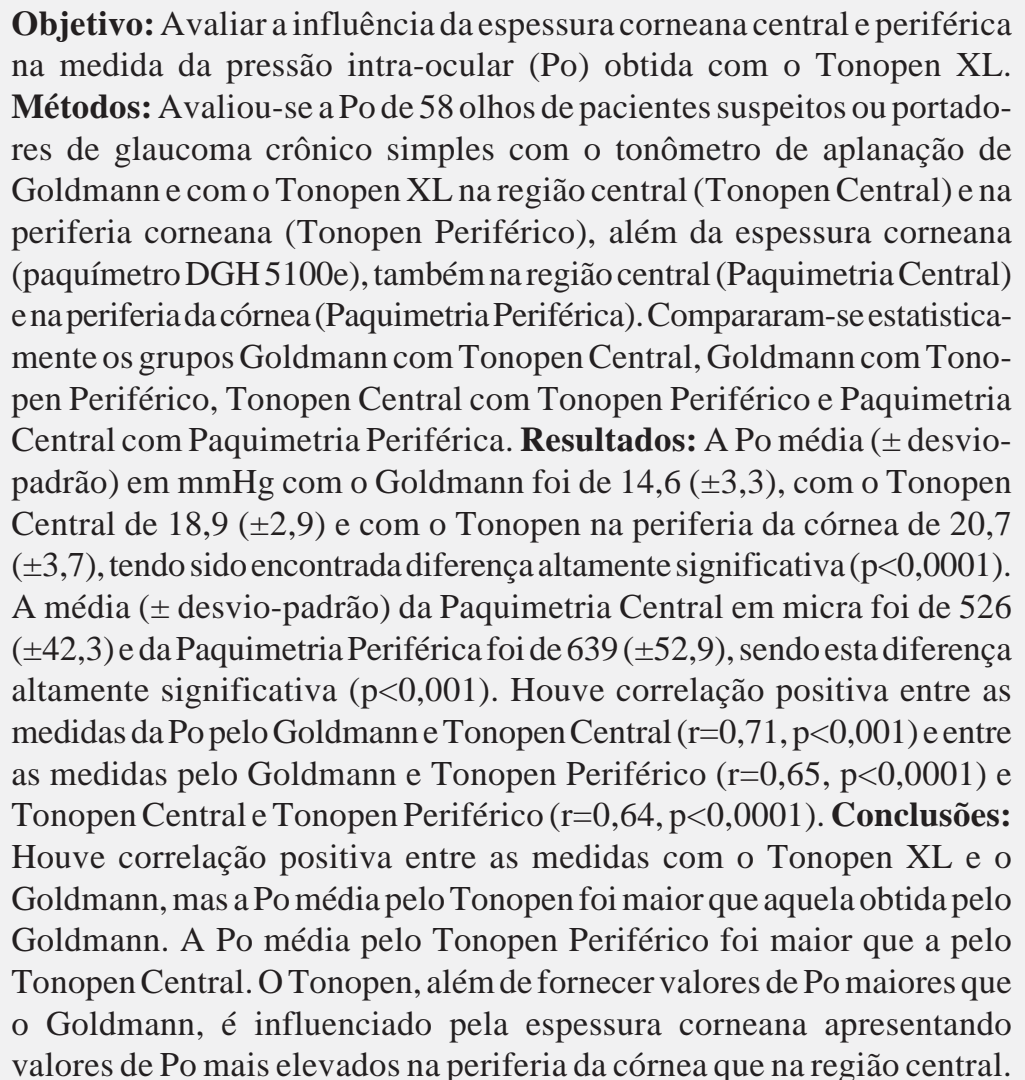 \\
\hline
\end{tabular}

Descritores: Córnea; Medidas; Pressão intra-ocular; Tonometria ocular

\section{INTRODUÇÃO}

O glaucoma é uma neuropatia óptica progressiva caracterizada pela lesão das células ganglionares da retina, que leva à perda de campo visual e, em seu estado final, à cegueira. Como a pressão intra-ocular (Po) é o principal e único fator de risco em que se pode atuar até o momento, tornase extremamente importante a sua avaliação correta.

A avaliação da Po é feita pelos tonômetros, sendo o mais amplamente utilizado o tonômetro de aplanação de Goldmann $(\mathrm{TAG})^{(1)}$, que mede a Po através do aplanamento de uma área central padronizada $\left(7,35 \mathrm{~mm}^{2}\right)$ da córnea. O Tonopen é um tonômetro portátil e computadorizado que também 
mede a Po pela aplanação da córnea operando pelo princípio de Mackay-Marg ${ }^{(2)}$ através de um mecanismo eletrônico.

Foi descoberto por Ehlers ${ }^{(3)}$ que a espessura corneana pode interferir com a medida obtida pelo tonômetro do Goldmann: córneas espessas tendem a superestimar o valor da Po e córneas finas, a subestimá-lo ${ }^{(4-6)}$. Teoricamente, talvez o Tonopen seria menos afetado pela espessura corneana do que o TAG, pois a área de aplanamento corneano é menor $\left(2,36 \mathrm{~mm}^{2}\right)$.

Este estudo tem como objetivo avaliar se a espessura da córnea influencia nas medidas feitas com o Tonopen, através da comparação entre a medida da Po realizada na região central da córnea (menos espessa) com a medida realizada na periferia corneana (mais espessa), utilizando-se as medidas obtidas com o tonômetro de Goldmann como padrão-ouro para a avaliação da Po.

\section{MÉTODOS}

Os pacientes foram selecionados entre aqueles atendidos no Serviço de Glaucoma, excluindo-se os portadores de lesões corneanas, entre fevereiro e agosto de 2004. Foram incluídos 58 olhos (30 pacientes) de pacientes suspeitos ou portadores de glaucoma crônico simples. Dois pacientes eram portadores de olho único. Foram considerados suspeitos de glaucoma os pacientes que apresentavam Po entre 19 a $25 \mathrm{mmHg}$ em tonometrias isoladas e/ou relação escavação/diâmetro do disco óptico de 0,6 a 0,8 e/ou assimetria da relação escavação/ diâmetro do disco óptico superior a 0,2 com campo visual normal. Os pacientes glaucomatosos apresentavam Po controlada sob medicação tópica antiglaucomatosa e alterações típicas de campo visual.

O protocolo de estudo foi previamente aprovado pelo Comitê de Ética do Departamento de Oftalmologia da Universidade Federal de Minas Gerais.

Os pacientes foram submetidos à tonometria com o Tonopen XL (Mentor Ophthalmics Inc.) nas regiões central e periférica (próxima ao limbo às 6 horas) da córnea, em seguida, à tonometria com o tonômetro de Goldmann (Haag-Streit) montado na lâmpada de fenda modelo Zeiss na região central da córnea, e por último, à paquimetria central e periférica (às 6 horas) com o paquímetro modelo DGH 5100e. Todas as medidas foram feitas pelo mesmo examinador (WOGA) no período da tarde entre 13 e 15 horas. Foram consideradas válidas as médias fornecidas pelo Tonopen calibrado de acordo com as instruções do fabricante e que apresentavam erro menor que $5 \%$. As medidas com o Tonopen foram feitas cobrindo-se a ponta do tonômetro com uma luva de látex trocada para cada paciente. Após as medidas da Po, foram feitas três medidas da espessura central e três da espessura periférica da córnea, considerando a média das três medidas em cada caso. As medidas de todos os parâmetros foram feitas sob prévia anestesia da córnea com proparacaína a $0,1 \%$. Para a medida da Po com o tonômetro de Goldmann, utilizou-se também a fluoresceína colírio a $0,25 \%$. Não foram feitas medidas da curvatura da córnea.
As informações colhidas foram armazenadas em banco de dados apropriado $^{(7)}$ e analisadas descritivamente e por meio de testes estatísticos (ANOVA), considerando-se o nível de significância de cinco por cento $(\mathrm{p}<0,05)$.

\section{RESULTADOS}

A tabela 1 mostra a média e desvio-padrão da Po obtida pelo Goldmann e pelo Tonopen Central e a tabela 2 a comparação entre a Po medida pelo Goldmann e Tonopen Periférico. A comparação entre os grupos mostrou uma diferença altamente

\begin{tabular}{|c|c|c|c|c|c|}
\hline & $\mathbf{N}$ & $\mathbf{x}$ & DP & $t$ & $\mathbf{p}$ \\
\hline Po Goldmann & 58 & 14,6 & 3,3 & \multirow{2}{*}{$-13,8$} & \multirow{2}{*}{$<0,0001$} \\
\hline Po Tonopen Central & 58 & 18,9 & 2,9 & & \\
\hline
\end{tabular}

\begin{tabular}{|c|c|c|c|c|c|}
\hline & $\mathbf{N}$ & $\mathbf{x}$ & DP & $t$ & p \\
\hline Po Goldmann & 58 & 14,6 & 3,3 & \multirow{2}{*}{$-15,5$} & \multirow{2}{*}{$<0,0001$} \\
\hline Po Tonopen Periférico & 58 & 20,7 & 3,7 & & \\
\hline
\end{tabular}

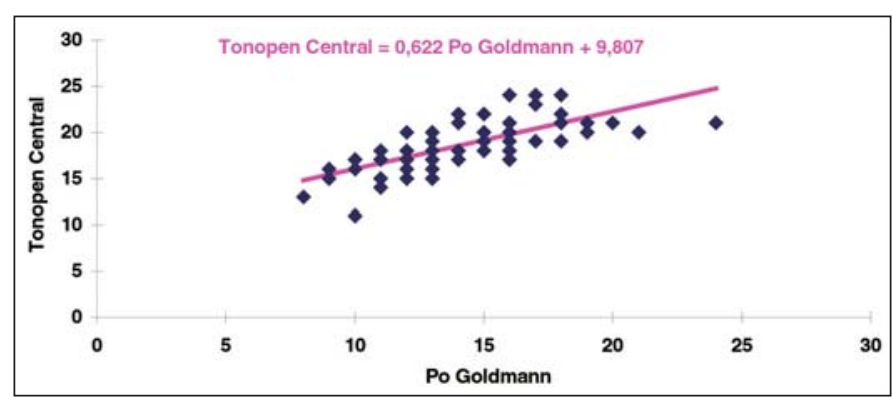

Gráfico 1 - Análise de regressão da Po Goldmann e Tonopen Central

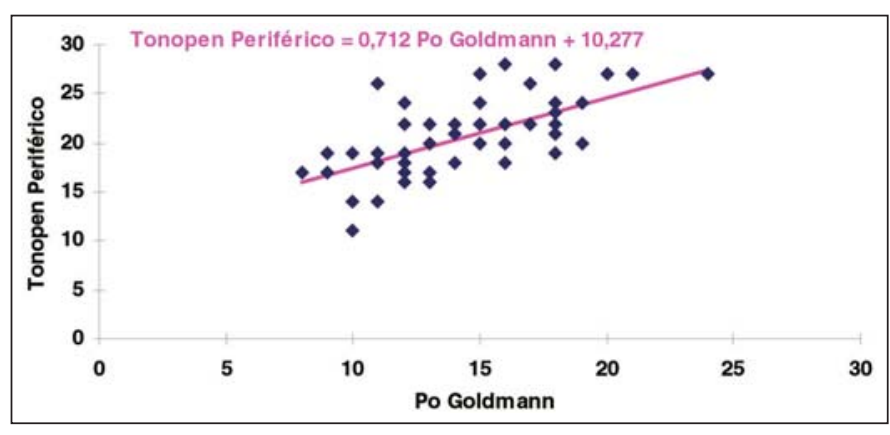

Gráfico 2 - Análise de regressão da Po Goldmann e Tonopen Periférico 
significativa, tanto entre a Po medida pelo tonômetro de Goldmann e pelo Tonopen Central quanto pelo Goldmann e Tonopen Periférico.

O gráfico 1 mostra a análise de regressão da medida da Po realizada pelo tonômetro de Goldmann e a medida realizada pelo Tonopen Central, e o gráfico 2, entre o tonômetro de Goldmann e o Tonopen Periférico. Observa-se maior dispersão dos valores no segundo gráfico em relação ao primeiro.

A tabela 3 mostra a comparação entre a Po medida pelo Tonopen Central e pelo Tonopen Periférico. Observamos diferença altamente significativa entre os dois grupos, sendo a média da Po pelo Tonopen periférico maior que a média pelo Tonopen Central.

O gráfico 3 mostra a análise de regressão entre a medida da Po realizada pelo Tonopen na região central (Tonopen Central) e na periferia da córnea (Tonopen Periférico). Nota se novamente maior dispersão dos valores em relação ao gráfico 1.

A tabela 4 mostra a comparação entre as médias da espessura corneana central (Paquimetria Central) e periférica ( $\mathrm{Pa}-$ quimetria Periférica), tendo sido constatada uma diferença altamente significativa entre elas.

O gráfico 4 mostra a análise de regressão entre a espessura central (Paq. Central) e periférica (Paq. Periférica) da córnea.

A tabela 5 mostra a média e desvio-padrão da variação entre a espessura corneana medida na região central da córnea (Paquimetria Central) e na região periférica (Paquimetria Periférica).

A tabela 6 mostra a comparação entre a variação da Po medida pelo Goldmann em relação à Po medida pelo Tonopen na região central da córnea (Variação Goldmann/Tonopen

\begin{tabular}{|c|c|c|c|c|c|}
\hline & $\mathbf{N}$ & $\mathbf{x}$ & DP & $\mathbf{t}$ & p \\
\hline Po Tonopen Central & 58 & 18,8 & 2,8 & \multirow{2}{*}{$-4,8$} & \multirow{2}{*}{$<0,0001$} \\
\hline Po Tonopen Periférico & 58 & 20,7 & 3,7 & & \\
\hline
\end{tabular}

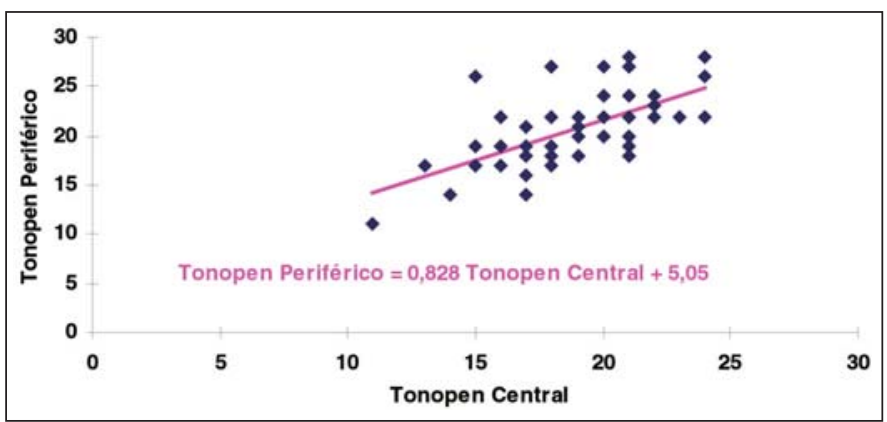

Gráfico 3 - Análise de regressão entre a medida da Po realizada pelo Tonopen na região central (Tonopen Central) e na periferia da córnea (Tonopen Periférico)
Central) e a variação da Po medida pelo Goldmann em relação à Po medida pelo Tonopen na região periférica da córnea (Variação Goldmann/Tonopen Periférico).

O gráfico 5 mostra a média das medidas da Po realizada pelo Tonopen na região central e periférica da córnea e a média da espessura corneana, também realizada na região central e periférica da córnea.

\section{DISCUSS ÃO}

Quando Goldmann e Schmidt ${ }^{(8)}$ criaram o tonômetro de aplanação deduziram que a espessura corneana poderia in-

\begin{tabular}{|c|c|c|c|c|c|}
\hline & $\mathbf{N}$ & $\mathbf{x}$ & DP & $\mathbf{t}$ & p \\
\hline Paquimetria Central & 58 & 526 & 42,3 & \multirow{2}{*}{$-25,5$} & \multirow{2}{*}{$<0,0001$} \\
\hline Paquimetria Periférica & 58 & 639 & 52,9 & & \\
\hline
\end{tabular}

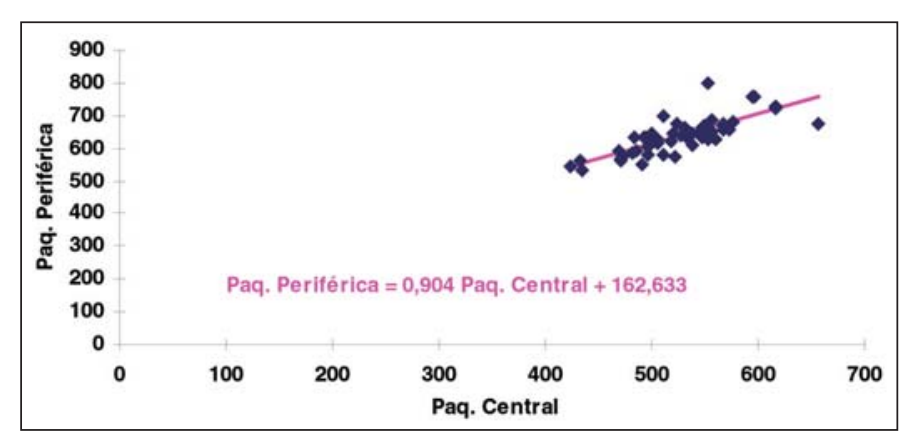

Gráfico 4 - Análise de regressão entre a espessura central (Paq. Central) e periférica (Paq. Periférica) da córnea

\begin{tabular}{|c|c|c|c|}
\hline & $\mathbf{N}$ & $\mathbf{x}$ & DP \\
\hline $\begin{array}{l}\text { Variação entre a Paquimetria Central } \\
\text { e a Paquimetria Periférica }\end{array}$ & 58 & 112 & 33,5 \\
\hline $\mathrm{N}=$ número de olhos; $\mathrm{x}=$ média; $\mathrm{DP}=$ desvio & & & \\
\hline
\end{tabular}

\begin{tabular}{|c|c|c|c|c|c|}
\hline & $\mathbf{N}$ & $x$ & DP & $t$ & $p$ \\
\hline $\begin{array}{l}\text { Variação Po } \\
\text { Goldmann/Tonopen } \\
\text { Central }\end{array}$ & 58 & 4,2 & 2,1 & \multirow[b]{2}{*}{$-4,5$} & \multirow[b]{2}{*}{$<0,0003$} \\
\hline $\begin{array}{l}\text { Variação Po } \\
\text { Goldmann/Tonopen } \\
\text { Periférico }\end{array}$ & 58 & 6,0 & 2,9 & & \\
\hline
\end{tabular}




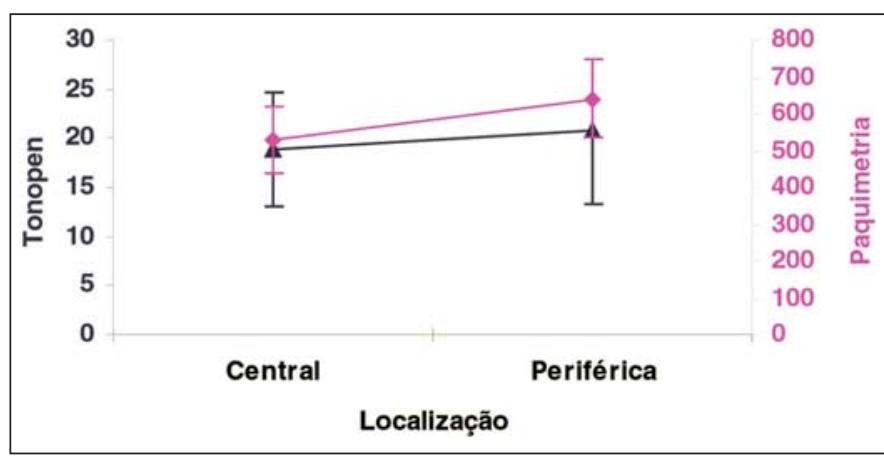

Gráfico 5 - Média das medidas da Po realizada pelo Tonopen e da espessura corneana realizada na região central e periférica da córnea

fluenciar na medida da Po, mas consideraram isto importante apenas em casos de patologia corneana, adotando uma paquimetria de aproximadamente 500 micra em córneas normais. Com o advento da paquimetria ultra-sônica ${ }^{(5-6)}$ observou-se que a variação da espessura corneana em córneas normais era muito maior do que Goldmann e Schmidt ${ }^{(8)}$ imaginaram.

Em estudo recente ${ }^{(9)}$, não foi encontrada diferença estatisticamente significativa na espessura central da córnea de suspeitos e portadores de glaucoma crônico simples.

Embora vários estudos já tenham tentado estabelecer a relação tonometria-paquimetria ${ }^{(4-6)}$, ainda não foi obtido um algoritmo capaz de correlacionar com precisão estas duas variáveis. Deste modo, é especialmente importante saber como as medidas de Po obtidas com diferentes tonômetros são afetadas por córneas de espessuras diferentes de tal forma que possamos fazer o raciocínio adequado para cada paciente suspeito ou portador de glaucoma.

Alguns estudos ${ }^{(10-11)}$ têm mostrado boa correlação entre as medidas da Po obtidas com o tonômetro de Goldmann e o Tonopen. Neste trabalho, encontramos correlação positiva ${ }^{(12)}$ entre os dois tonômetros para as medidas da Po pelo Goldmann e o Tonopen Central $(r=0,71 ; \mathrm{p}<0,0001)$ e para as medidas pelo TC e o TP $(r=0,65 ; \mathrm{p}<0,0001)$. Apesar disso, é necessário ter cautela na análise das medidas dos valores da Po pois os valores obtidos com o Tonopen na região central da córnea foram, em média, $4,2 \mathrm{mmHg}$ superiores aos obtidos com o tonômetro de Goldmann e 6,0 $\mathrm{mmHg}$ superiores aos obtidos quando a medida com o Tonopen foi realizada na periferia da córnea (Tabela 6). Além disso, o coeficiente de correlação é um dado genérico e não serve para comparar amostras com diferenças significativas, sendo melhor, nesses casos, utilizar outros testes (como o t pareado), como já foi demonstrado anteriormente ${ }^{(13)}$. Considerando que o tonômetro de Goldmann é atualmente o padrão-ouro na medida da Po, o Tonopen forneceria medidas de Po maiores que a pressão intra-ocular real (medida pelo tonômetro de Goldmann), o que pode ter implicações importantes em pacientes com glaucoma. Possivelmente, as diferenças encontradas neste trabalho em relação a outros ${ }^{(10-11,14)}$ sejam devidas às diferenças de metodologias. No presente estudo, todas as medidas da Po foram realizadas pelo mesmo examinador e primeiro com o Tonopen. Para alguns autores ${ }^{(1)}$, quando o tonômetro de Goldmann é usado em primeiro lugar os valores de Po obtidos com o Tonopen são subestimados enquanto que quando o Tonopen é usado inicialmente (como foi feito neste trabalho) há uma superestimação dos valores da Po. Optamos por usar primeiramente o Tonopen e acreditamos ser essa a metodologia correta por dois motivos. Inicialmente, porque o tonômetro de Goldmann aplana uma maior área da córnea $\left(7,35 \mathrm{~mm}^{2}\right)$ o que, segundo alguns autores ${ }^{(1)}$, pode exercer maior influência na medida da Po do que no caso de iniciarmos a medida pelo Tonopen com o qual a área de aplanação da córnea $\left(2,36 \mathrm{~mm}^{2}\right)$ é cerca de 3,1 vezes menor. Em segundo lugar, porque para a medida correta com o tonômetro de Goldmann há necessidade da instilação de colírio de fluoresceína a $0,25 \%$, não utilizado na medida da Po com o Tonopen.

Como o Tonopen necessita de uma pequena área de aplanação para a medida da Po, teoricamente, seria mais indicado para o estudo da Po em olhos com irregularidades corneanas $^{(11)}$. De fato, em algumas situações, a realização da tonometria de aplanação de Goldmann é impossível e o Tonopen pode ser de grande valia nesses casos, devendo-se ficar atentos, no entanto, para a superestimativa do valor da Po em relação ao que seria encontrado com o tonômetro de Goldmann.

Quando se considera a espessura corneana e o Tonopen, alguns autores não encontraram influência ${ }^{(11)}$ ou encontraram pouca influência ${ }^{(14)}$ da espessura sobre a medida da Po. Por outro lado, outros autores ${ }^{(15)}$ não só consideraram que a espessura corneana influencia a medida da Po realizada pelo Tonopen como enfatizaram a necessidade de correção na leitura obtida em córneas espessas. Analisando-se a tabela 3, constata-se um acréscimo no valor da leitura da Po obtida pelo Tonopen ao se fazer a medida na periferia da córnea em relação à sua região central; como a Po não se altera de uma região para a outra, é possível deduzir que algum fator esteja atuando no sentido de elevar falsamente a medida da leitura da Po na periferia da córnea. Também é visível o aumento da espessura do centro da córnea para a sua periferia (Tabela 4). Podemos, levando-se em consideração que já é fato que a espessura corneana interfere na medida da Po feita pelo tonômetro de Goldmann, deduzir que essa variável também interfere na medida realizada pelo Tonopen. Portanto, o presente estudo corrobora as conclusões de outros ${ }^{(15)}$, pois também encontramos evidências de que a espessura corneana altera a medida da Po com o Tonopen; há um aumento do valor da leitura da Po quando a medida com o Tonopen é realizada na periferia da córnea, onde a espessura corneana é maior que na região central. Isto sugere que em córneas mais espessas a medida da Po com o Tonopen pode ser maior do que a encontrada em córneas com menor espessura. Entretanto, novos estudos, com amostras maiores, fazem-se necessários, para melhor correlacionar tonometria-paquimetria nos diversos tipos de tonômetros existentes na atualidade.

\section{CONCLUSÕES}

No presente estudo, avaliamos a influência da espessura corneana central e periférica na medida da Po com o Tonopen, 
utilizando a tonometria de Goldmann como padrão-ouro. Concluímos que:

1) Apesar da correlação positiva entre as medidas da Po obtidas com os dois tonômetros na região central da córnea, o Tonopen forneceu valores de Po maiores (em média, 4,2 $\mathrm{mmHg}$ ) que os obtidos pelo tonômetro de Goldmann. Na periferia da córnea, os valores de Po obtidos com o Tonopen foram ainda maiores (em média, $6,0 \mathrm{mmHg}$ ).

2) A maior diferença de valor da Po entre o tonômetro de Goldmann e o Tonopen foi encontrada quando se realizou a medida com o Tonopen na periferia da córnea (onde a espessura corneana é maior), confirmando que o aumento da espessura corneana também influencia na medida da Po com o Tonopen.

3) Em casos de dúvida na avaliação da Po com o Tonopen, recomenda-se medir a espessura da córnea.

\section{ABSTR ACT}

Purpose: To assess the influence of central and peripheral corneal thickness on the IOP measurement by Tonopen. Methods: The IOP of 58 eyes from suspected and glaucomatous patients was evaluated by Goldmann's tonometry (GT) and, by Tonopen XL on the central (TC) and peripheral (TP) regions of the cornea. Using the DGH 5100e pachymeter, we also measured the corneal thickness in its central (CCT) and peripheral (at 6:00 h) regions (PCT). Patients with any corneal changes were excluded. The mean values of all measurements was comparatively analyzed (GT x TC; GT x TP; TC x TP; CCT x PCT) by the paired $t$ test and by regression and correlation analysis. Results: The means ( \pm standard deviation) of IOP obtained with GT, TC and TP were respectively $14.6( \pm 3.3) \mathrm{mmHg}$; $18.9( \pm 2.9) \mathrm{mmHg}$ and $20.7( \pm 3.7) \mathrm{mmHg}$ with a statistical highly significant difference $(\mathrm{p}<0.001)$. The means $( \pm$ standard deviation) of central and peripheral corneal thickness were respectively $526( \pm 42.3)$ and $639( \pm 52.9) \mu \mathrm{m}$, also with a statistical highly significant difference $(\mathrm{p}<0.001)$. The correlation coefficients $(r)$ between the measurements were $0.71(\mathrm{p}<0.001)$ for GT and TC; $0.65(\mathrm{p}<0.001)$ for GT and TP and $0.64(\mathrm{p}<0.001)$ for TC and TP. Conclusions: There was a positive correlation between the measurements performed with Tonopen XL and Goldmann's tonometer at the central region of the cornea and the mean IOP was higher with Tonopen XL. The mean IOP obtained by TP was higher than that obtained by TC. These data showed that Tonopen XL, besides providing higher IOP readings than those with Goldmann's tonometer, is influenced by the corneal thickness presenting higher IOP readings in the periphery than in the central region of the cornea.

Keywords: Cornea; Measures; Intraocular pressure; Tonometry, ocular

\section{REFERÊNCIAS}

1. Iester M, Mermoud A, Achache F, Roy S. New Tonopen XL: comparison with the Goldmann tonometer. Eye. 2001;15(Pt 1):52-8.

2. Wirt H, Draeger J, Rumberger E, Deutsch C, Dauper J. [Comparative studies of the calibration of new electronic automatic tonometers]. Fortschr Ophthalmol. 1989;86(4):403-6. German.

3. Ehlers N. On corneal thickness and intraocular pressure. II. A clinical study on the thickness of the corneal stroma in glaucomatous eyes. Acta Ophthalmol (Copenh). 1970;48(6):1107-12.

4. Meirelles SHS, Stohler NSF, Mariano MP, Yamane R, Yamane Y. Espessura corneana no glaucoma de pressão normal. Rev Bras Oftalmol. 2001;60(1):56-64.

5. Herndon LW, Weizer JS, Stinnett SS. Central corneal thickness as a risk factor for advanced glaucoma damage. Arch Ophthalmol. 2004;122(1):17-21.

6. Brandt JD, Beiser JA, Kass MA, Gordon MO. Central corneal thickness in the Ocular Hypertension Treatment Study (OHTS). Ophthalmology. 2001;108(10): 1779-88.

7. Dean AG, Dean JA, Coulombier D, Brendel KA, Smith DC, Burton AH, et al. Epi Info Version 6: a Word processing, database and statistics program for epidemiology on microcomputers. Atlanta (USA): Centers for Disease Control and Prevention; 1994.

8. Goldmann H, Schmidt T. [Applanation tonometry]. Ophthalmologica. 1957; 134(4):221-42. German.

9. Cronemberger S, Calixto N, Costa LT, Soares FM. Corneal thickness and daily curve of intraocular pressure in suspected and glaucomatous patients. Arq Bras Oftalmol. 2005;68(2):185-8.

9. Bandyopadhyay M, Raychaudhuri A, Lahiri SK, Schwartz EC, Myatt M, Johnson GJ. Comparison of Goldmann applanation tonometry with the Tonopen for measuring intraocular pressure in a population-based glaucoma survey in rural West Bengal. Ophthalmic Epidemiol. 2002;9(3):215-24.

10. Rao VJ, Gnanaraj L, Mitchell KW, Figueiredo FC. Clinical comparison of ocular blood flow tonometer, Tonopen, and Goldmann applanation tonometer for measuring intraocular pressure in postkeratoplasty eyes. Cornea. 2001;20(8): 834-8.

11. Bhan A, Browning AC, Shah S, Hamilton R, Dave D, Dua HS. Effect of corneal thickness on intraocular pressure measurements with the pneumotonometer, Goldmann applanation tonometer, and Tono-Pen. Invest Ophthalmol Vis Sci. 2002;43(5):1389-92.

12. Rodrigues PC. Bioestatística. 3a ed. Niterói: Edu FF; 2002.

13. Calixto N, Bonfioli A, Waisberg Y. Vergleichende Untersuchung der Tonometer von Mackay-Marg, Perkins und Draeger im Verhältnis zum GoldmannTonometer. In: Glaukom-Symposion. Würzburg; 1974. p.172-81.

14. Mok KH, Wong CS, Lee VW. Tono-Pen tonometer and corneal thickness. Eye. 1999;13(Pt 1):35-7.

15. Dohadwala AA, Munger R, Damji KF. Positive correlation between Tonopen intraocular pressure and central corneal thickness. Ophthalmology. 1998; 105(10):1849-54

Nos artigios enviados para publicação, o nome dos autores e suas afiliações devem estar completos. Isso facilitará a indexação e os links com as bases de dados e o CV Lates. 